\title{
Geometrical scaling of prompt photons in heavy ion colli- sions
}

\author{
Michat Praszałowicz ${ }^{1, *}$ \\ ${ }^{1}$ M. Smoluchowski Institute of Physics, Jagiellonian University, S. Łojasiewicza 11, 30-348 Kraków, \\ Poland
}

\begin{abstract}
We discuss geometrical scaling (GS) for the prompt (direct) photons produced in heavy ion collisions. To this end we first introduce the concept of GS and illustrate its emergence on the example of charged particles. Next, we analyse direct photon data from RHIC and from the LHC. We show that the data support the hypothesis of GS in terms of participant number related to different centrality classes. We also study GS at different energies, however a more detailed study will be possible only when data for at least three different energies from one experiment will be available and also when we use the data obtained from the collisions of large systems $(\mathrm{Cu}+\mathrm{Cu}, \mathrm{Au}+\mathrm{Au}, \mathrm{Pb}+\mathrm{Pb})$ and small systems $(p+p, d+A u, p+A u)$.
\end{abstract}

\section{Introduction}

It is now widely accepted that in high energy scattering the bulk features of the produced particles spectra give access to the properties of the initial state. Especially, in the small Bjorken $x$ kinematical region, where the initial hadrons consist predominantly from the overoccupied gluonic cloud, a phenomenon called geometrical scaling (GS) arrises. Geometrical scaling has been first observed in the inclusive deep inelastic electron-proton scattering (DIS) [1], where the reduced cross section, essentially $F_{2}\left(x, Q^{2}\right) / Q^{2}$, which is in principle a function of two variables $x$ and $Q^{2}$, depends in fact on only one scaling variable $\tau=Q^{2} / Q_{\text {sat }}^{2}(x)$. The saturation scale

$$
Q_{\mathrm{sat}}^{2}(x)=Q_{0}^{2}\left(x / x_{0}\right)^{-\lambda}
$$

is directly proportional to the gluon density in the proton, and $x_{0}$ and $Q_{0}$ are fixed parameters of the order of $10^{-3}$ and $1 \mathrm{GeV} / c$, respectively. Exponent $\lambda \approx 0.33$ is a nonperturbative dynamical quantity following from the properties of the (non-linear) QCD evolution equation, however its numerical value has to be fixed from the data (for review see Ref. [2]).

The properties of the saturated gluon densities should have an impact on the particles produced in proton-proton collisions. Indeed, adopting a so called parton-hadron duality [3], one can argue that the bulk properties of the gluon spectra are shared by charged particles that are eventually detected experimentally. In particular they exhibit GS in the small $x$ region (i.e. large energy $\sqrt{s}$ and moderate $p_{\mathrm{T}}$ ) $[4,5]$. However, multiplicity spectra scale with a somewhat lower power $\lambda$ than the one extracted from DIS. We have argued that for pp scattering, where one does not control the overlap area transverse to the center of mass

\footnotetext{
*e-mail: michal@if.uj.edu.pl
} 
reaction axis, the quantity that scales with $\lambda \approx 0.33$ is the differential cross section, rather than multiplicity [6]. This should be contrasted with the heavy ion (HI) reactions, when the transverse overlap area is controlled by an appropriate choice of centrality classes.

The fact that GS exhibited by the gluons is transferred to the final state particles, which are created in the nonperturbative hadronization process, which undergo final state interactions, which are being produced by resonance decays, is by no means obvious. This is even more so in the case of HI collisions where the quark-gluon plasma (QGP) is created and undergoes hydrodynamical evolution. Nevertheless, following Refs. [7, 8], we will show in the next section that GS in charged particles spectra is indeed present even in this case.

From this point of view direct photons (by definition photons that do not originate from hadronic decays) are an excellent probe of the initial state of HI collisions since they do not interact strongly while passing through the quark-gluon plasma. However, photons are produced from quarks (through annihilation and Compton scattering), and therefore they do not probe the overoccupied gluonic cloud directly. To this end one employs the Color Glass Condensate (CGC) effective theory where the initial gluonic CGC evolves into an intermediate state called glasma, which is a strongly interacting not thermalised QGP [2]. Quarks are produced in the thermalisation process, and - if there are no other mass scales around their distribution should exhibit geometrical scaling ${ }^{1}$ in variable $\tau=p_{\mathrm{T}}^{2} / Q_{\mathrm{sat}}^{2}(x)$. Similarly, the photon spectra should scale as well. In order to validate this scenario one should check if the available photon data exhibit geometrical scaling. The first step in this direction has been undertaken in Ref. [12] where the functional form of the photon spectra has been assumed to be of the form $p_{\mathrm{T}}^{-n}$ (see also Refs. [9-11]). Here, following Refs. [4-8], we shall employ model independent method of ratios to analyse GS of photon spectra, which is done in Sect. 3. But first, in Sect. 2, we shortly recall analysis of charged particle spectra already reported in Refs. [7, 8]. We conclude in Sect. 4.

\section{Charged particles}

By geometrical scaling [1] of charged particles in hadronic collisions we mean that the multiplicity distributions are well described - up to the logarithmic correction of the running coupling constant - by a universal function $F(\tau)[4,5]$ :

$$
\frac{1}{S_{\perp}} \frac{d N_{\mathrm{ch}}}{d \eta d^{2} p_{\mathrm{T}}}=F(\tau)
$$

of the scaling variable

$$
\tau=\frac{p_{\mathrm{T}}^{2}}{Q_{\mathrm{sat}}^{2}} .
$$

Saturation scale (for particles produced in mid rapidity region) reads therefore

$$
Q_{\text {sat }}^{2}=Q_{0}^{2}\left(\frac{p_{\mathrm{T}}}{W x_{0}}\right)^{-\lambda} \text {. }
$$

Here $W=\sqrt{s}$ is the scattering energy and for the reference we take $x_{0}=10^{-3}$ and $Q_{0}=1$ $\mathrm{GeV} / c$. Parameter $S_{\perp}$ is a transverse area, which for heavy ion collisions corresponds to geometrical overlap of the colliding nuclei at given impact parameter $b$ [13]. HI data are usually divided into centrality classes that select events within certain range of impact parameter $b$.

\footnotetext{
${ }^{1}$ Detailed description of the photon production mechanism in glasma is beyond scope of this report; we refer the reader to Refs. [9-11].
} 
In this case both transverse area $S_{\perp}$ and the saturation scale $Q_{\text {sat }}^{2}$ acquire additional dependence on centrality that is characterized by an average number of participants $N_{\text {part }}$. We have $[12,13]$ :

$$
S_{\perp} \sim N_{\text {part }}^{\delta} \text { and } Q_{\text {sat }}^{2} \sim N_{\text {part }}^{\delta / 2} .
$$

where one typically assumes $\delta=2 / 3$, which follows from the collision geometry. Therefore in $\mathrm{HI}$ collisions

$$
\frac{1}{N_{\text {evt }}} \frac{d N_{\mathrm{ch}}}{N_{\text {part }}^{\delta} 2 \pi p_{\mathrm{T}} d \eta d p_{\mathrm{T}}}=\frac{1}{Q_{0}^{2}} F(\tau)
$$

and the scaling variable $\tau$ takes the following form:

$$
\tau=\frac{p_{\mathrm{T}}^{2}}{N_{\text {part }}^{\delta / 2} Q_{0}^{2}}\left(\frac{p_{\mathrm{T}}}{W}\right)^{\lambda} .
$$

Taking $x_{0}=10^{-3}$ we have that the energy $W$ should be expressed in $\mathrm{TeV}$, whereas $p_{\mathrm{T}}$ in $\mathrm{GeV}$. The analysis presented here will be only qualitative and we shall not fine tune the scaling exponent $\lambda$ keeping it fixed at $\lambda=0.3$.

In this section we shall analyse ALICE data on PbPb collisions at $2.76 \mathrm{TeV}$ [14] and also earlier data from RHIC from STAR [15, 16] and PHENIX [17, 18] collaborations at 200 and $130 \mathrm{GeV}$ per nucleon respectively. Centrality classes together with participants numbers are given in Table 1.

Table 1. Centrality classes and the corresponding numbers of participants in heavy ion experiments analysed in this paper. Energies per nucleon in $\mathrm{TeV}$ are displayed next to the experiment name. Bold face entries in blue (color on-line) show classes of similar number of participants analysed in the text.

\begin{tabular}{|rr|rr|rr|rr|}
\hline \multicolumn{2}{|c|}{ ALICE 2.76} & \multicolumn{2}{|c|}{ STAR 0.2 \& 0.13} & \multicolumn{2}{|c|}{ PHENIX 0.2} & \multicolumn{2}{|c|}{ PHENIX 0.13} \\
centrality & \multicolumn{1}{|c|}{$N_{\text {part }}$} & centrality & $N_{\text {part }}$ & centrality & \multicolumn{1}{c|}{$N_{\text {part }}$} & centrality & $N_{\text {part }}$ \\
\hline $0-5 \%$ & 383 & & & & & & \\
$\mathbf{5 - 1 0 \%}$ & $\mathbf{3 3 0}$ & $\mathbf{0 - 5 \%}$ & $\mathbf{3 5 0}$ & $\mathbf{0 - 1 0 \%}$ & $\mathbf{3 5 2 . 2}$ & $\mathbf{0 - 5 \%}$ & $\mathbf{3 4 8}$ \\
& & $5-10 \%$ & 296 & & & $5-15 \%$ & 271 \\
$10-20 \%$ & 261 & $10-20 \%$ & 232 & $10-20 \%$ & 234.6 & & \\
$\mathbf{2 0 - 3 0 \%}$ & $\mathbf{1 8 6}$ & $\mathbf{2 0 - 3 0 \%}$ & $\mathbf{1 6 5}$ & $\mathbf{2 0 - 3 0 \%}$ & $\mathbf{1 6 6 . 6}$ & $\mathbf{1 5 - 3 0 \%}$ & $\mathbf{1 8 0}$ \\
$30-40 \%$ & 129 & $30-40 \%$ & 115 & $30-40 \%$ & 114.2 & & \\
$40-50 \%$ & 85 & & & $40-50 \%$ & 74.4 & $30-60 \%$ & 79 \\
$50-60 \%$ & 53 & $40-60 \%$ & 62 & $50-60 \%$ & 45.5 & & \\
$\mathbf{6 0 - 7 0 \%}$ & $\mathbf{3 0}$ & $\mathbf{6 0 - 8 0 \%}$ & $\mathbf{2 0}$ & $\mathbf{6 0 - 7 0 \%}$ & $\mathbf{2 5 . 7}$ & $\mathbf{6 0 - 8 0 \%}$ & $\mathbf{1 9 . 5}$ \\
$70-80 \%$ & 15.8 & & & $70-80 \%$ & 13.4 & & \\
& & & & $80-92 \%$ & 6.3 & $80-92 \%$ & 5.5 \\
\hline
\end{tabular}

In Fig. 1 we plot multiplicity distributions from all three experiments displayed in Table 1, for different centrality classes and - in the case of STAR and PHENIX - for both scattering energies. First in the left panel multiplicity distributions are plotted as functions of $p_{\mathrm{T}}$ and then in the right panel as functions of scaling variable $\sqrt{\tau}(7)$. We see that different distributions from the left panel coincide over some range of $\sqrt{\tau}$ when scaled according to Eq. (6). For clarity in Fig. 1 we have used only every second centrality class of ALICE and PHENIX@200 GeV data.

We can see from Fig. 1 that, indeed, heavy ion data scale according to (6) up to $\sqrt{\tau} \sim 1.8$ approximately. The quality of scaling is, however, not as good as in the case of pp scattering [5]. One could perhaps improve the quality of GS by tuning the exponent $\lambda$ in the definition 
of $\tau$ (7). We have decided to keep $\lambda$ constant for the purpose of present analysis because of the systematic differences between the data from different collaborations. The data has been taken in the rapidity intervals that are different in different experiments, also the partition of the data into centrality classes varies from one experiment to another as can be seen from Table 1.
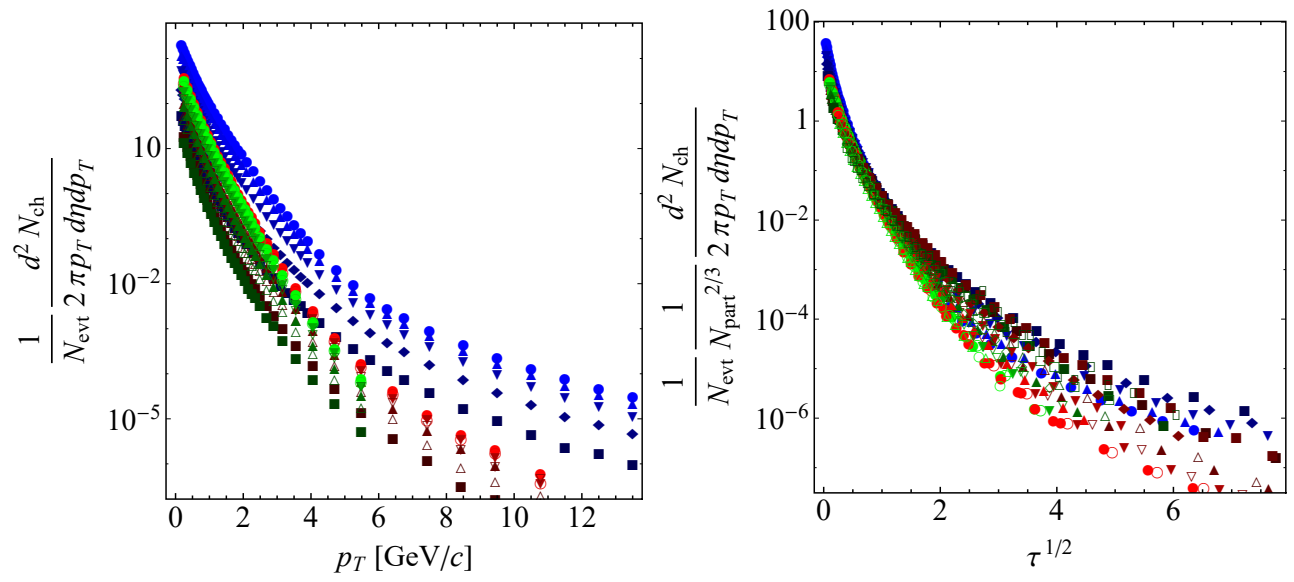

Figure 1. Illustration of geometrical scaling in heavy ion collisions at different energies and different centrality classes. Left panel shows charged particle distributions from ALICE [14], STAR [15, 16] and PHENIX $[17,18]$ plotted as functions of $p_{\mathrm{T}}$. In the right panel the same distributions are scaled according to Eq. (6). Symbols used here are the same as in Figs. 2-3.
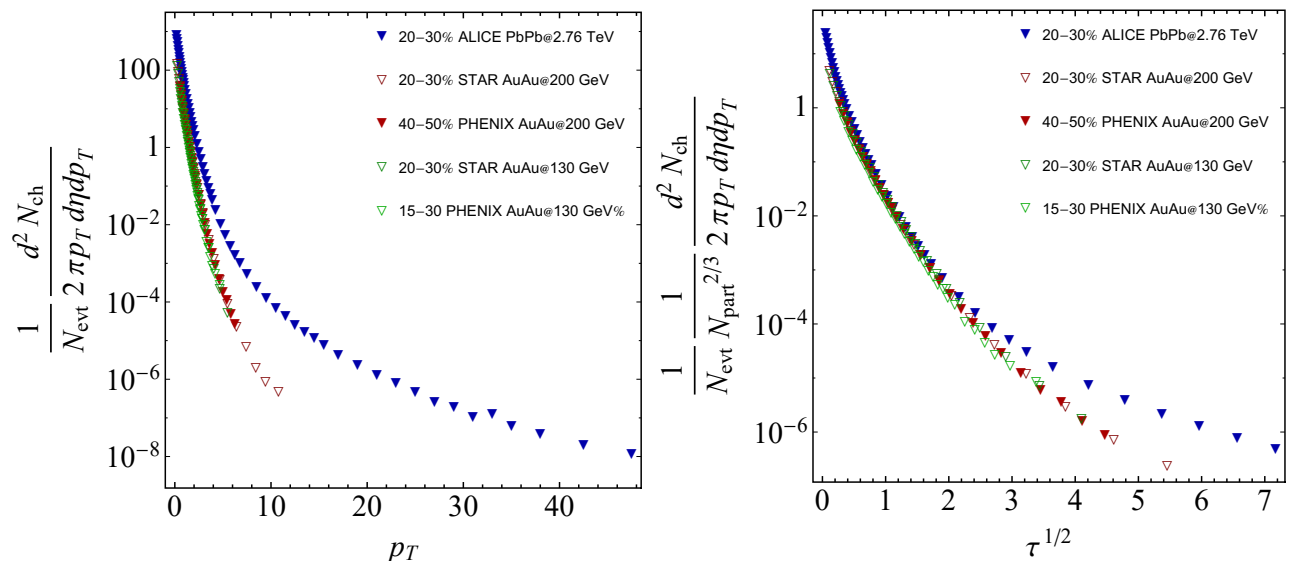

Figure 2. Illustration of geometrical scaling in heavy ion collisions at different energies for mid centrality classes corresponding to $n_{\text {part }}=165-186$. Left panel shows charge particle distributions from ALICE [14], STAR [15, 16] and PHENIX [17, 18] plotted as functions of $p_{\mathrm{T}}$. In the right panel the same distributions are scaled according to Eq. (6).

It is interesting to test now the quality of GS separately in dependence on energy and on centrality (i.e. on $N_{\text {part }}$ ). Let us first discuss scaling with energy by selecting centrality classes that correspond to similar number of participants in all three experiments. For illustration we 
plot in in Fig. 2 multiplicity distributions with $n_{\text {part }}=165-186$ (second blue row in Table 1). We see rather good scaling up to $\sqrt{\tau} \approx 2$. The plots for other centralities look very much the same.

For fixed scattering energy $W$ equation (6) relates distributions of different participant number. We shall now examine the quality of this scaling by plotting multiplicity distributions at the same energy but different centrality classes. In Fig. 3 we plot ALICE data at $2.76 \mathrm{GeV}$. Plots for RHIC energies are very similar, and one can say that generally centrality scaling is of worse quality than the energy scaling discussed above.
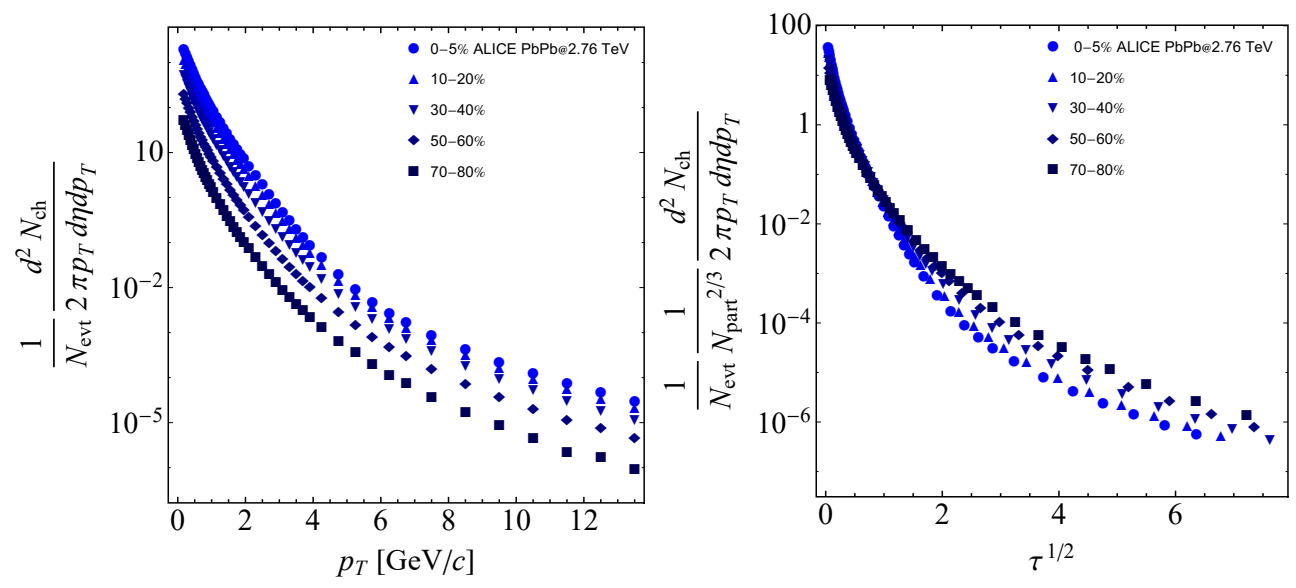

Figure 3. Illustration of geometrical scaling in heavy ion collisions at fixed ALICE [14] energy of 2.76 $\mathrm{TeV}$ for different centrality classes. Left panel shows charge particle distributions plotted as functions of $p_{\mathrm{T}}$. In the right panel the same distributions are scaled according to Eq. (6).

\section{Photons}

In this paper we shall analyse the following data sets on direct photons: PHENIX [19, 20] $\mathrm{Au}+\mathrm{Au} @ 200 \mathrm{GeV}$ with the following centrality classes $0-20 \%\left(N_{\text {part }}=277.5\right), 20-40 \%$ $\left(N_{\text {part }}=135.6\right), 40-60 \%\left(N_{\text {part }}=56.0\right), 60-92 \%\left(N_{\text {part }}=12.5\right)$ and ALICE $^{2}[21,22]$ $\mathrm{Pb}+\mathrm{Pb} @ 2.76 \mathrm{TeV}: 0-20 \%\left(N_{\text {part }}=308\right), 20-40 \%\left(N_{\text {part }}=157\right)$ and $40-80 \%\left(N_{\text {part }}=45.7\right)$. More recent PHENIX data [23] reported this year has not been available at the time of preparing this manuscript.

\section{1 $N_{\text {part }}$ scaling}

Let us first examine the $N_{\text {part }}$ dependence of geometrical scaling for the ALICE data [22]. These spectra ale plotted in the left panel of Fig. 4, where we have included points with $p_{\mathrm{T}} \leq 10 \mathrm{GeV} / c$. In the right panel the same spectra ale plotted after rescaling according to (6) for $\delta=2 / 3$. We see that to a very good accuracy all three spectra coincide. This result should be contrasted with the charged particle scaling shown in Fig 3, which is of much worse quality. The same analysis is illustrated in Fig. 5 for PHENIX data [19, 20].

\footnotetext{
${ }^{2}$ Special thanks to Jacek Otwinowski for providing us with the pertinent values of $N_{\text {part }}$.
} 

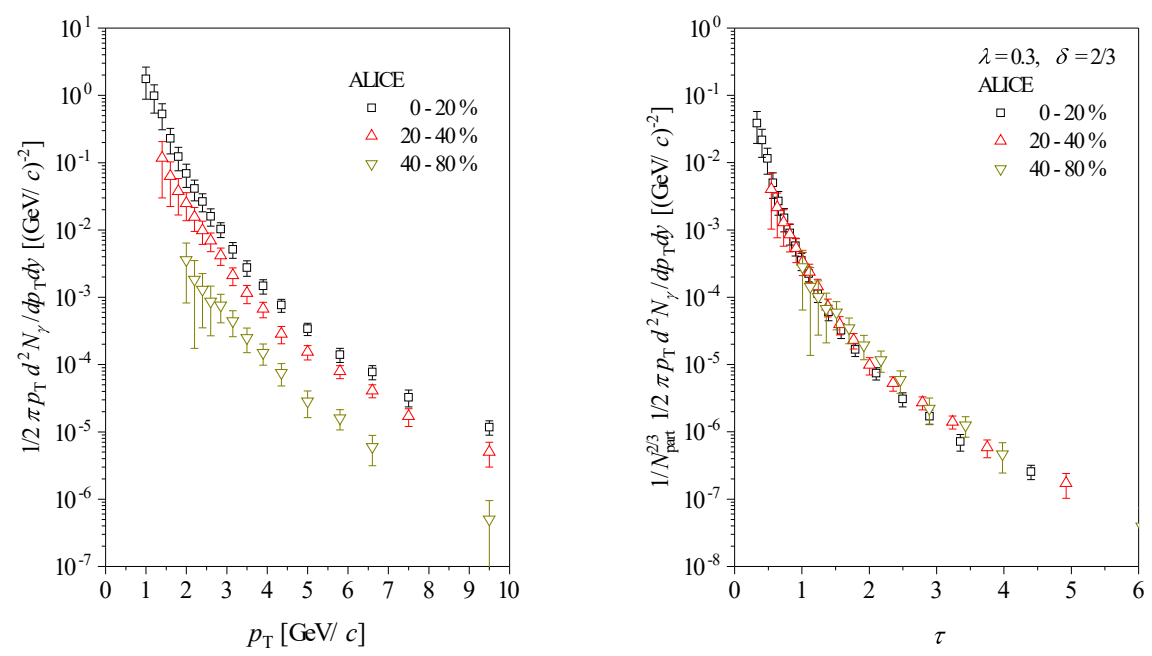

Figure 4. Illustration of $N_{\text {part }}$ geometrical scaling of the $\gamma$ yields in heavy ion collisions for different centrality classes for ALICE PbPb data. Left panel: transverse momentum spectra of direct photons at three centrality classes: $0-20 \%$ (black squares), $20-40 \%$ (red up-triangles) $40-80 \%$, (dark green downtriangles). Right panel: scaled spectra for $\delta=2 / 3$. Data from Ref. [22].
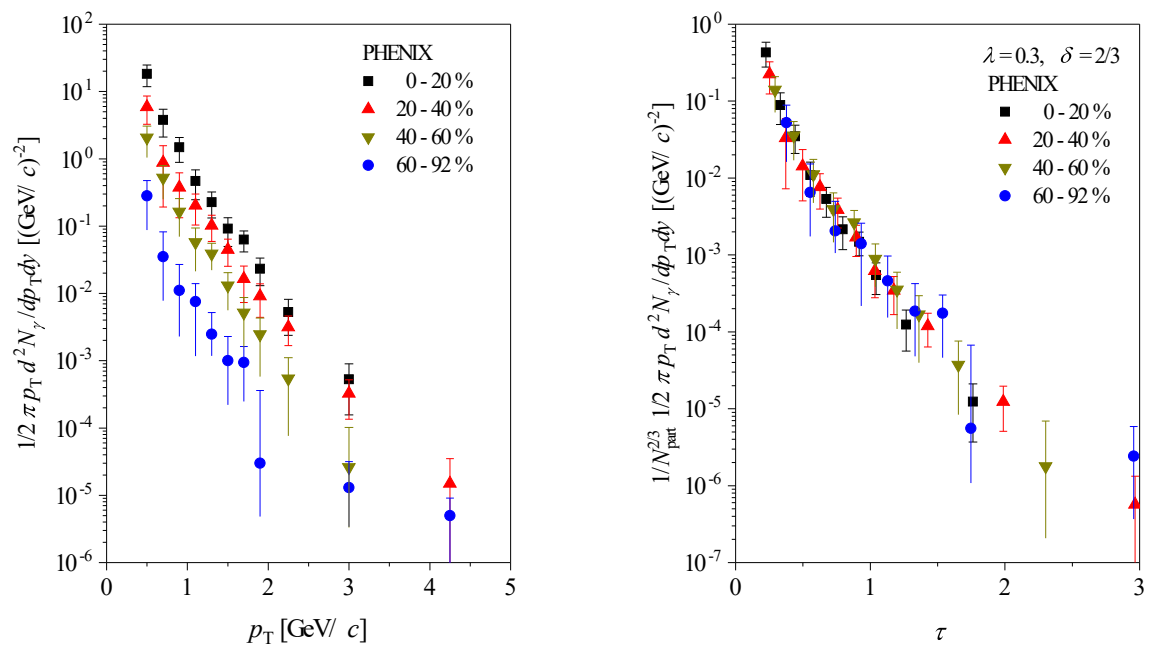

Figure 5. Illustration of $N_{\text {part }}$ geometrical scaling of the $\gamma$ yields in heavy ion collisions for different centrality classes for PHENIX AuAu data at $200 \mathrm{GeV}$ [20]. Left panel: transverse momentum spectra of direct photons at four centrality classes: $0-20 \%$ (black squares), 20-40\% (red up-triangle) $40-80 \%$ (dark green down-triangles), 6-92\% (blue circles). Right panel: scaled spectra for $\delta=2 / 3$. Lower error bars without end caps have been arbitrarily shortened for better visibility.

In order to examine the quality of $N_{\text {part }}$ scaling we construct the ratios of the scaled spectra at different centralities $c_{1}$ and $c_{2}, c_{3}$ or $c_{4}$

$$
R_{c_{1} / c_{2,3}}(\tau)=\frac{1}{N_{1 \text { part }}^{\delta}} \frac{d N_{\gamma}^{(1)}}{N_{\text {evt }} 2 \pi p_{\mathrm{T}} d \eta d p_{\mathrm{T}}}(\tau) / \frac{1}{N_{2,3 \text { part }}^{\delta}} \frac{d N_{\gamma}^{(2,3)}}{N_{\text {evt }} 2 \pi p_{\mathrm{T}} d \eta d p_{\mathrm{T}}}(\tau)
$$



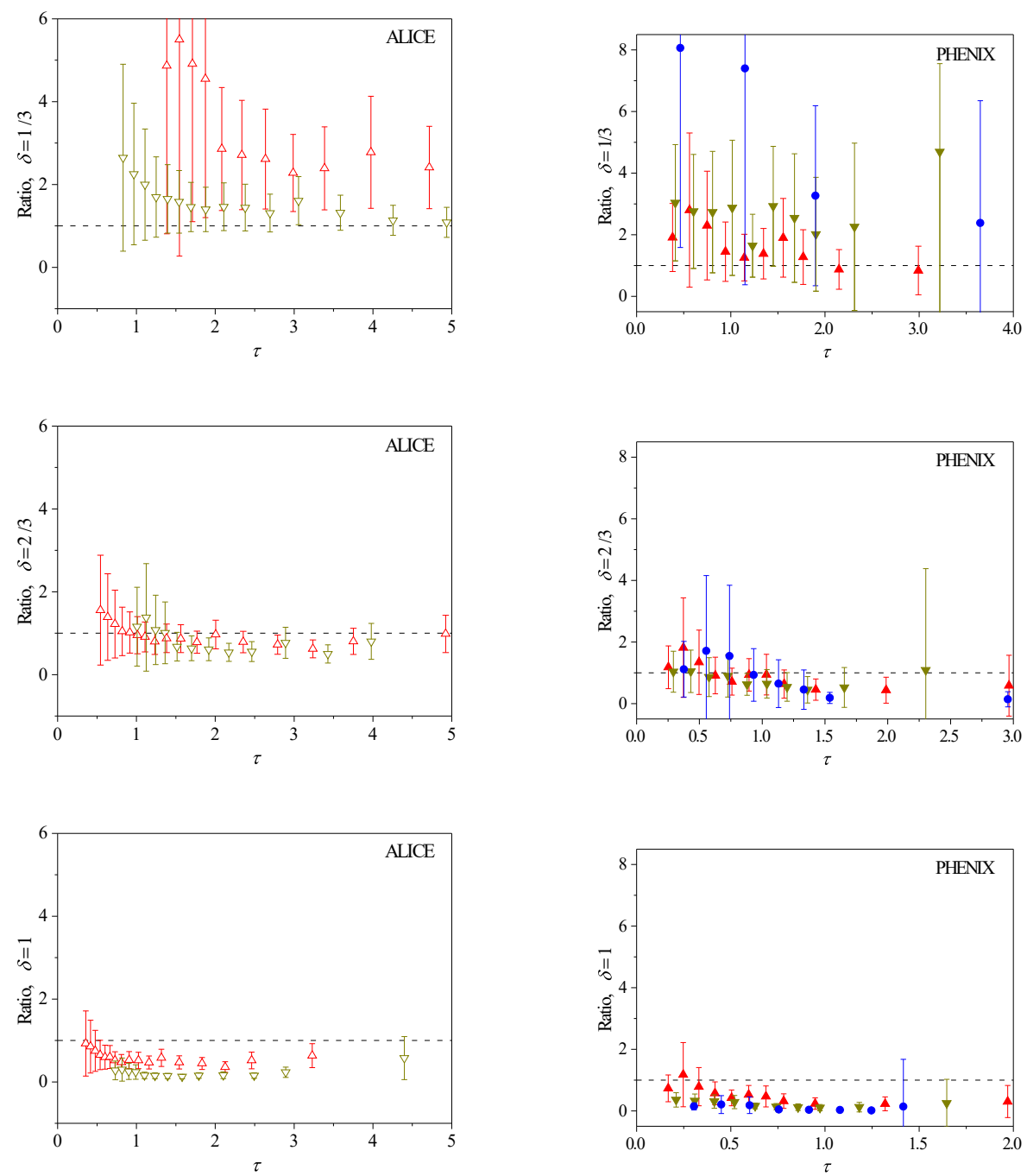

Figure 6. Illustration of geometrical scaling of the $\gamma$ yields in heavy ion collisions at different centrality classes. Left panel: ALICE. Red triangles correspond to $R_{c_{1} / c_{2}}$ and dark-grren ones to $R_{c_{1} / c_{3}}$. Right panel: PHENIX. Red triangles points correspond to $R_{c_{1} / c_{2}}$, dark-green ones to $R_{c_{1} / c_{3}}$ and blue ones to $R_{c_{1} / c_{4}}$. Upper plot $\delta=1 / 3$, middle plot $\delta=2 / 3$ and the lower plot $\delta=1$.

and plot them for different $\delta$ as functions $\tau$ in Fig. 6. In the left panel we plot ALICE data where red up-triangles points correspond to $c_{2}=20-40 \%$ and the dark green down-triangles to $c_{3}=40-80 \%$. Geometrical scaling is achieved when $R_{c_{1} / c_{2}} \approx R_{c_{1} / c_{3}} \sim 1$. As one can see from Fig. 6 , this happens indeed for $\delta \approx 2 / 3$. The same ratios for PHENIX data are plotted in the right panel of Fig. 6 where red triangles correspond to $c_{2}=20-40 \%$, dark-green ones to $c_{3}=40-60 \%$ and blue ones to $c_{4}=60-92 \%$. 


\subsection{Energy scaling}

Having established that GS is indeed achieved for $\delta=2 / 3$ (or very close to $2 / 3$ ), we can now test energy scaling, i.e. $\lambda$ dependence of GS. Unfortunately, the quality of ratios, similar to the ones defined in Eq. (8), but for different energies rather than centralities, is very poor as compared to (8). This is because the data from different experiments suffer from systematic uncertainties, like different rapidity ranges, different definition of centrality classes, etc. Therefore in Fig. 7 we simply plot spectra both for ALICE and PHENIX in terms of scaling variable $\tau$ defined in Eq. (7) for two different choices of $\lambda$ : 0.2 in left panel and 0.3 in right panel. We see that it is hard to decide for which $\lambda$ GS is better (with logarithmic accuracy). This shows that it is of importance to have data at different energies (at least three) from one experiment where the systematic uncertainties mentioned above cancel.
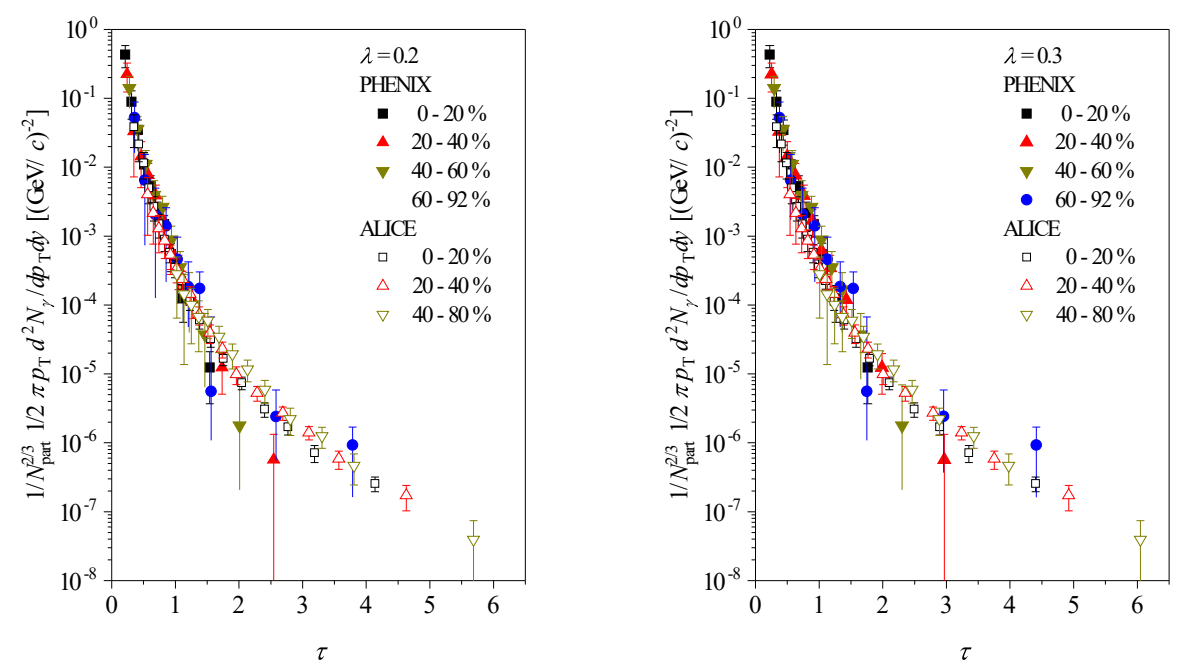

Figure 7. Illustration of geometrical scaling of the $\gamma$ yields in heavy ion collisions for different centrality classes for PHENIX AuAu data at $200 \mathrm{GeV}$ [20] and Alice data at 2.76 TeV [22]. Left panel: $\lambda=0.2$, right panel $\lambda=0.3$. Lower error bars without end caps have been arbitrarily shortened for better visibility.

\section{Conclusions}

Geometrical scaling is the property of the overoccupied gluonic cloud that is characterised by the new dynamical scale, called saturation scale. If this is the only energy scale in a given kinematical region, then by a simple argument of dimensional analysis particle spectra should depend only on the ratio of the transverse momentum to this scale. This phenomenon has been first observed in inclusive DIS and then also in hadronic collisions. In the latter case the emergence of GS is by no means obvious due the the final state interactions, resonance decays, confinement, etc. Nevertheless GS is observed in pp scattering and also in HI collisions.

Photons that have weak final state interactions (they are rather insensitive to the QGP medium) and are free from the confinement effects seem to be a much better probe of the initial state than charged particles. This is, however, not entirely true, since photons do not 
couple to gluons, and therefore the fact that they still exhibit GS provides a positive test of quark production mechanism in glasma.

The analysis presented here should be carried out on the recent PHENIX data [23] including also the data obtained from the collisions of different systems $(\mathrm{Cu}+\mathrm{Cu}$ [24], $\mathrm{Au}+\mathrm{Au}$ [25], $\mathrm{Pb}+\mathrm{Pb}$ ) and small systems (p+p, d+Au, p+Au) - for review see Ref. [26].

\section{Ackonwledgenments}

The author acknowledges very useful discussions with Larry McLerran and Vladimir Khachatryan. This research has been supported the Polish National Science Centre grant 2014/13/B/ST2/02486.

\section{References}

[1] A. M. Stasto, K. J. Golec-Biernat and J. Kwiecinski, Phys. Rev. Lett. 86, 596 (2001)

[2] L. McLerran, Acta Phys. Pol. B 41 (2010) 2799

[3] Y. L. Dokshitzer, V. A. Khoze and S. I. Troian, J. Phys. G 17, 1585 (1991)

[4] L. McLerran and M. Praszalowicz, Acta Phys. Pol. B 41, 1917 (2010)

[5] L. McLerran and M. Praszalowicz, Acta Phys. Pol. B 42, 99 (2011)

[6] M. Praszalowicz and A. Francuz, Phys. Rev. D 92, 074036 (2015)

[7] M. Praszalowicz, PoS DIS 2015, 084 (2015)

[8] M. Praszalowicz, Acta Phys. Pol. B Proc. Supp. 8399 (2015)

[9] M. Chiu, T. K. Hemmick, V. Khachatryan, A. Leonidov, J. Liao and L. McLerran, Nucl. Phys. A 900, 16 (2013)

[10] L. McLerran and B. Schenke, Nucl. Phys. A 946, 158 (2016)

[11] V. Khachatryan, B. Schenke, M. Chiu, A. Drees, T. K. Hemmick and N. Novitzky, Nucl. Phys. A 978, 123 (2018)

[12] C. Klein-Bösing and L. McLerran, Phys. Lett. B 734, 282 (2014)

[13] D. Kharzeev, E. Levin and M. Nardi, Nucl. Phys. A 747, 609 (2005)

[14] B. Abelev et al. [ALICE Collaboration], Phys. Lett. B 720, 52 (2013)

[15] J. Adams et al. [STAR Collaboration], Phys. Rev. Lett. 91, 172302 (2003)

[16] C. Adler et al. [STAR Collaboration], Phys. Rev. Lett. 89, 202301 (2002)

[17] S. S. Adler et al. [PHENIX Collaboration], Phys. Rev. C 69, 034910 (2004)

[18] K. Adcox et al. [PHENIX Collaboration], Phys. Rev. Lett. 88, 022301 (2002)

[19] A. Adare et al. [PHENIX Collaboration], Phys. Rev. Lett. 104, 132301 (2010)

[20] A. Adare et al. [PHENIX Collaboration], Phys. Rev. C 91, 064904 (2015)

[21] M. Wilde [ALICE Collaboration], Nucl. Phys. A 904-905, 573c (2013)

[22] J. Adam et al. [ALICE Collaboration], Phys. Lett. B 754, 235 (2016)

[23] A. Adare et al. [PHENIX Collaboration], arXiv:1805.04084 [hep-ex]

[24] A. Adare et al. [PHENIX Collaboration], Phys. Rev. C 98, 054902 (2018)

[25] S. Afanasiev et al. [PHENIX Collaboration], Phys. Rev. Lett. 109, 152302 (2012)

[26] V. Khachatryan [PHENIX Collaboration], arXiv:1812.01841 [nucl-ex] 